\title{
Preparation of a Novel One-Armed Anti-c-Met Antibody with Antitumor Activity Against Hepatocellular Carcinoma
}

This article was published in the following Dove Press journal:

Drug Design, Development and Therapy

\author{
Yanxin Yin $\mathbb{1}^{1,2, *}$ \\ jia Guo ${ }^{1,2, *}$ \\ Fei Teng $\mathbb{( D}^{1,2}$ \\ Lihua $\mathrm{Yu}\left(\mathbb{D}^{2}\right.$ \\ Yun Jiang (D) ${ }^{2}$ \\ Kun Xie $\mathbb{D i D}^{3}$ \\ Ming Jiang ${ }^{1,2}$ \\ Jianmin Fang ${ }^{\mathrm{I}-3}$
}

'Department of Neurology, Shanghai Tongji Hospital, Tongji University School of Medicine, Shanghai 200065, People's Republic of China; ${ }^{2}$ Biomedical Research Center, Tongji University Suzhou Institute, Suzhou, Jiangsu 215101 , People's Republic of China; ${ }^{3}$ School of Life Science and Technology, Tongji University, Shanghai 200092, People's Republic of China

*These authors contributed equally to this work
Correspondence: Ming Jiang Biomedical Research Center, Tongji University Suzhou Institute, Building 2, 198 Jinfeng Road, Wuzhong District, Suzhou, Jiangsu 2I5I0I, People's Republic of China

Tel +86 5I 2 660I 6586

Email jiangm@tongji.edu.cn

Jianmin Fang

School of Life Science and Technology,

Tongji University, 1239 Siping Road,

Shanghai 200092, People's Republic of

China

Tel +8621 65982878

Email jfang@tongji.edu.cn
Introduction: Antibody-based c-mesenchymal-epithelial transition factor (c-Met) inhibition is a promising strategy for hepatocellular carcinoma (HCC) treatment, but the intrinsic agonistic activity of the anti-c-Met antibody limits its application in drug development. Constructing a monovalent one-armed antibody has been reported to be an effective way to create an inhibitory anti-c-Met antibody.

Materials and methods: In the present study, a novel monovalent one-armed anti-c-Met antibody was constructed using the knobs-into-holes technology, and its inhibitory effects against HCC and the underlying mechanisms were explored.

Results: The one-armed anti-c-Met antibody blocked the hepatocyte growth factor (HGF)/ c-Met interaction and the subsequent signal transduction, including phosphorylation of c-Met, Grb2-associated binding protein 1(Gab-1), extracellular regulated protein kinases 1/ 2(Erk1/2), and Akt, also referred to as protein kinase B (PKB) in HCC cell line HepG2. Furthermore, the autocrine stimulation of HepG2 cell proliferation and HGF-induced HCC cell migration were strongly inhibited by the one-armed anti-c-Met antibody. In addition, the antibody also reduced the HGF-induced proliferation and tube formation of human umbilical vein endothelial cells (HUVECs). Treating HepG2-bearing mice with the one-armed antic-Met antibody significantly inhibited the tumor growth in the xenograft nude mouse model. Conclusion: The one-armed anti-c-Met antibody derived from the full-length bivalent antic-Met antibody might serve as a potential antitumor agent against HCC.

Keywords: antitumor, c-Met, hepatocellular carcinoma, knobs into holes, monovalent antibody, targeted therapy

\section{Introduction}

Hepatocellular carcinoma (HCC) is a highly lethal disease with a rising incidence. Around 746,000 people die of HCC across the world annually, making it the second leading cause of cancer death. ${ }^{1}$ It is difficult to diagnose HCC early. Therefore, its highly malignant features and rapid progression result in poor outcome and prognosis. Despite adopting the Barcelona Clinic Liver Cancer strategy in HCC treatment, the overall 5-year survival remains poor at $20 \%{ }^{2}$ Therefore, novel treatment strategies for HCC, especially for late-stage HCC, are urgently needed.

The multifunctional growth factor scatter factor/hepatocyte growth factor (SF/ HGF) and its receptor c-mesenchymal-epithelial transition factor (c-Met) participate in cancer progression by enhancing several cancer hallmarks. ${ }^{3-6}$ In the case of solid tumors, hypoxia can enhance HGF/c-Met expression, ${ }^{7,8}$ which facilitates 
angiogenesis and stimulates the growth, locomotion, and invasion of cancer cells. In HCC, the protein level of c-Met is $25-100 \%$ higher than that in normal liver, ${ }^{9}$ suggesting a promising target for HCC. The interruption of the $\mathrm{HGF} / \mathrm{c}-\mathrm{Met}$ axis is widely adopted as an anticancer strategy. Some c-Met inhibitors, such as tivantinib, golvatinib, and cabozantinib, have been described to interfere with the c-Met signaling pathway. ${ }^{10,11}$ However, the side effects and resistance of these inhibitors remain unresolved. Antibody-based approaches to interrupt the HGF/ c-Met interaction have been explored in the last few years. ${ }^{12}$ However, the intrinsic agonistic activity of the bivalent monoclonal antibody (mAb) makes it difficult to develop a promising anti-c-Met $\mathrm{mAb}$ that can antagonize the dimerization and autophosphorylation of the receptor tyrosine kinase (RTK). ${ }^{13,14}$

A monovalent one-armed anti-c-Met antibody was produced using the knobs-into-holes technology. The binding affinity of the antibody to the extracellular part of c-Met was preserved, but the ability to induce c-Met dimerization was abolished. Onartuzumab (OA-5D5, MetMAb) is a onearmed humanized anti-c-Met antibody derived from an agonistic antibody 5D5. Onartuzumab has shown antitumor activity in multiple human tumor xenograft models, ${ }^{15-17}$ including lung, colon, breast, stomach, and brain. However, it was not previously evaluated in HCC models.

In the present study, a monovalent anti-c-Met antibody was successfully prepared using the knobs-into-holes technology to potently inhibit HCC growth, migration, and angiogenesis in vitro and in vivo. Further, several mechanisms by which this antibody inhibited HCC progression were identified.

\section{Materials and Methods}

The lentiviral vectors for the expression of the monovalent antibody against the human c-mesenchymal-epithelial transition factor were constructed in the laboratory. The primers were synthesized by GENEWIZ Bio. (Suzhou, China) Phusion High-Fidelity Polymerase Chain Reaction (PCR) Master Mix, restriction enzymes, and T4 DNA ligase were procured from NEW ENGLAND BioLabs, Inc. (MA, USA). Bacteria competent cells, gel extraction kit, and plasmid Miniprep kit were purchased from Axygen Scientific, Inc. (CA, USA).

\section{Antibodies and Reagents}

The antibodies used for immunoblotting analysis were as follows: Phospho-Gab1 (Tyr307) Antibody\#3234, Gab1
Antibody\#3232, Phospho-Met (Tyr1234/1235) (D26) XP rabbit $\mathrm{mAb} \# 3077$, Met (D1C2) XP rabbit mAb\#8198, Akt (pan) (C67E7) rabbit mAb\#4691, phospho-Akt (Ser473) (D9E) XP rabbit mAb\#4060, p44/42 Mitogen-activated protein kinase (MAPK) (Erk1/2) (137F5) rabbit mAb\#4695, phospho-p44/42 MAPK (Erk1/2) (Thr202/ Tyr204) (D13.14.4E) XP rabbit mAb\#4370, beta-actin (13E5) rabbit mAb\#4970, and anti-rabbit immunoglobulin $\mathrm{G}$, horseradish peroxidase (HRP)-linked Antibody $\# 7074$ (all from Cell Signaling Technology, Inc., MA, USA). Beta-actin was used as the internal standard in this study.

Bivalent anti-c-Met antibodies were produced using the hybridoma method. BALB/c mice were immunized with the extracellular domain of human c-Met linked to His tag (c-Met ECD-His), and parental monoclonal antibodies were selected for their ability to bind to c-Met and inhibit the HGF/c-Met interaction. The monovalent one-armed anti-c-Met antibody was produced using the knobs-intoholes technology. ${ }^{17}$ The anti-c-Met antibody heavy chain was modified to encode the T366S:L368A:Y407V mutations, and the $\mathrm{Fc}$ fragment was modified to contain the T366W mutation. The light chain, mutated heavy chain, and Fc fragment were co-transfected into HEK-293 cells. The products were purified with Ni Sepharose 6 Fast Flow (\#17-5318-06, GE Healthcare., MA, USA).

\section{Antibody Candidate Screening}

The binding affinity of anti-c-Met antibodies was evaluated using enzyme-linked immunosorbent assay (ELISA) for the reactivity to c-Met ECD-His. The selected candidates were further evaluated using fluorescence-activated cell sorting (FACS) for the affinity to living cells. The antitumor activity of the antibodies was evaluated using a ligand-binding assay with ELISA for the ability to compete with HGF, Western blot for the inhibitory activity of c-Met phosphorylation, a 2-(4-Iodophenyl)-3-(4-nitrophenyl)-5-(2,4-disulfophenyl)2H-tetrazolium, monosodium salt (WST-8)-Cell Counting Kit-8 (CCK-8) for the anti-cell growth activity, Transwell assay for the anti-migration activity and tube formation assay for the anti-angiogenesis activity.

\section{Cell Culture}

HepG2 and HEK293 cells were obtained from the American Type Culture Collection (VA, USA). The cells were incubated with Dulbecco's modified Eagle's Medium (DMEM) (Gibco Co., Ltd, USA.) containing 10\% fetal bovine serum (FBS, qualified, Australia\#10099-141; Gibco Co., Ltd) in a humidified incubator in the presence 
of $5 \% \mathrm{CO}_{2}$ at $37^{\circ} \mathrm{C}$. Human umbilical vein endothelial cells (HUVECs) were obtained from the Allcells Co., Ltd. (Shanghai, China). The cells were incubated with HUVEC complete medium (\#H-004, Allcells Co., Ltd.).

\section{Establishment of the c-Met Overexpressing and Depleted Cells}

The genes of the extracellular domain ligated with the transmembrane domain of the human c-Met (prrl-c-Met-TM) and RNAi sequence of TCAACTTCTTTGTAGGCAATA (prrlHep G2-shc-Met) were cloned into the lentivirus plasmid. Chinese hamster ovary (CHO) and Hep G2 cells were transiently transfected with prrl-c-Met-TM and prrl-shc-Met respectively, using Lipofectamine 3000 (Thermo Fisher Scientific Co., Ltd, USA). The cells were harvested for flow cytometry analysis after $48-72 \mathrm{~h}$ of incubation.

\section{Enzyme-Linked Immunosorbent Assay}

The recombinant fusion protein encoding the c-Met ECDHis was plated onto a 96-well ELISA plate (Corning Co., Ltd. USA) at a concentration of $1 \mu \mathrm{g} / \mathrm{mL}$. After overnight incubation at $4^{\circ} \mathrm{C}$, the plates were washed with phosphatebuffered saline (PBS) containing 0.05\% Tween-20, blocked with $1 \%$ BSA in PBS, and probed with recombinant HGF $(0.1 \mu \mathrm{g} / \mathrm{mL})$ or the anti-c-Met antibodies for $1 \mathrm{~h}$, followed by incubation with an HRP-conjugated anti-human HGF antibody (American Qulex, CA, USA) or an HRP-conjugated secondary antibody. The reaction was visualized by adding 3,3',5,5'-tetramethylbenzidine (Beyotime Biotechnology, Shanghai, China), and the absorbance was read at $450 \mathrm{~nm}$ using a Bio-Rad iMark microplate reader.

\section{Cell Proliferation Assay}

The cells were seeded in the 96-well plates and treated with HGF, antibody, or other reagents for $72 \mathrm{~h}$. After the treatment, the cells were counted using a 2-(4-iodophenyl)-3-(4-nitrophenyl)-5-(2,4-disulfophenyl)-2H-tetrazolium, monosodium salt (WST-8)-Cell Counting Kit-8 (Dojindo Laboratories, Kumamoto, Japan). The average absorbance of the treated cells was detected using a Bio-Rad iMark Microplate Reader.

\section{Cell Migration Assay}

Transwell chambers were purchased from BD Biosciences (MA, USA). HepG2 cells $\left(2.5 \times 10^{4}\right.$ cells per well) were seeded into the upper chamber of the Transwell inserts and maintained at $37^{\circ} \mathrm{C}$ throughout the experiment. The cells were allowed to migrate toward the lower chamber in a medium containing either an HGF or an antibody for $24 \mathrm{~h}$. The cell migration was evaluated after the crystal violet staining by counting cells in six randomly chosen fields.

\section{Tube Formation Assay}

Matrigel (Corning Inc., MA, USA) was dissolved at $4^{\circ} \mathrm{C}$. Aliquots $(100 \mu \mathrm{L} /$ well) were added to the 96 -well plates, which were incubated at $37^{\circ} \mathrm{C}$ overnight. HUVECs, resuspended at a concentration of $3 \times 10^{4} / 100 \mu \mathrm{L}$ in a medium containing HGF, antibody, or other reagents, were added to each Matrigel-coated well. After 18-20 h of incubation at $37^{\circ} \mathrm{C}$, the cell capillary-like structure formation of HUVECs was assessed by microscopy, and the images of each well were obtained using a light microscope.

\section{Flow Cytometric Analysis}

The binding affinity of the antibody to the cell surface expressing c-Met was determined by flow cytometry analysis. Approximately $1 \times 10^{6}$ cells were harvested from a monolayer culture, washed, and incubated with a saturating amount $(10 \mu \mathrm{g} / \mathrm{mL})$ of primary antibody in PBS (pH 7.4) with 2\% FBS (staining buffer) for $30 \mathrm{~min}$ on ice. The cells were then washed and stained with an Alexa Fluor 488-conjugated goat anti-mouse IgG1 secondary antibody (diluted 1:100; Invitrogen, USA) for $15 \mathrm{~min}$ on ice. The cells were subsequently incubated with a fluoresceinconjugated $\mathrm{mAb}$ for $30 \mathrm{~min}$ on ice. The cell suspension was washed three times with PBS and then analyzed using a FACS Calibur flow cytometer (BD Immunocytometry Systems, NJ, USA).

\section{Immunoblotting Analysis}

Total proteins were extracted from the treated cells using radioimmunoprecipitation assay (RIPA) lysis buffer (Beyotime Biotechnology; Cat No. P0013B). The concentrations were measured using a bicinchoninic acid (BCA)Protein Assay Kit (Beyotime Biotechnology; Cat. P0012, Lot No. 040717170810). After electrophoresis, the proteins were transferred onto an Immobilon-P Transfer Membrane (Millipore Corporation, USA; Cat No. IPVH00010, Lot No. K3EA8230FK) and finally detected using a Supersignal West Femto Maximum Sensitivity Substrate (Thermo Scientific, USA; Cat No. 34096, Lot No. TC263090).

\section{Xenograft Mouse Model}

Eighteen 6- to 8-week-old BALB/c nude mice were purchased from SLRC Laboratory Animal Co., Ltd. (Shanghai, China) and housed in the specific-pathogen-free animal 
facility in Tongji University (Shanghai, China) for 1 week before the experiments. The mice had ad libitum access to filtered tap water in a specific-pathogen-free animal room under supervision. The facility staff monitored animal health care daily and took care of commercial feed supply, cleaning of cages and beddings, animal observation and report, and so forth. The animal care and animal experiments were approved by the Tongji University Laboratory Animal Care and Use Committee and performed according to the committee's guidelines. All surgeries were performed under sodium pentobarbital anesthesia, and all efforts were made to minimize suffering. HepG2 cells $\left(5 \times 10^{6}\right.$ cells/mouse $)$ with Matrigel (Corning, NY, USA) were subcutaneously inoculated into the right flanks of mice $(n=6$ /group, 18 mice in total) to allow tumor isografts to grow to an approximate size of $50 \mathrm{~mm}^{3}$ [tumor volume $=($ length $\times$ width $\times$ height $) / 2$ ] The mice were randomly assigned to treatment and control groups and received intraperitoneal injections of vehicle and 2E6 (5 and $10 \mathrm{mg} / \mathrm{kg}$ ) twice a week for up to 4 months. Despite applying humane endpoints in the present study, no mice became severely ill or injured prior to the experimental endpoint. All mice were euthanized by the intraperitoneal injection of sodium pentobarbital $(500 \mathrm{mg} / \mathrm{kg}$, SigmaAldrich, MO, USA) at the end of the experiments.

\section{Statistical Analysis}

Data were statistically analyzed using GraphPad Prism 6 (Graph Pad, CA, USA). Differences between the antibodytreated and the control-treated groups were analyzed using the Student's $t$-test. A $P$ value of less than 0.05 was considered to represent a significant difference.

\section{Results}

\section{Generation and Characterization of the One-Armed Anti-c-Met Antibody}

Monoclonal antibodies specific for c-Met were generated by immunizing $\mathrm{BALB} / \mathrm{c}$ mice with a recombinant fusion protein encoding the extracellular domain of human c-Met linked to His tag (c-Met ECD-His) and screened for the competitive inhibitory capacity against HGF/c-Met interaction in a ligand-binding assay. The highly specific lead antibody 1H9D6, which inhibited the HGF binding to c-Met, was selected for further recombinant construction. Since the bivalent antibody caused ligand-independent RTK activation, some monovalent antibody structures were invented to develop an inhibitory RTK antibody, but its short half-life in vivo limited their broad application. The knobs-into-holes technology was employed to construct a one-armed antic-Met antibody to overcome the short half-life of the monovalent antibody, by introducing hole mutations (T366S, L368A, and Y407V) in the Fc of a full-length heavy chain and a knob mutation (T366W) in the single Fc fragment (Figure 1A). These mutations were expected to facilitate heterodimerization between a full-length heavy chain and a single Fc fragment and promote the assembly of the monovalent antibody. The one-armed anti-c-Met antibody 2E6 was generated in an eukaryotic cell line HEK293, which coexpressed the chimeric 1H9D6 light chain, a hole containing full-length heavy chain, and a knob containing Fc chain. The denaturing sodium dodecyl sulfate-polyacrylamide gel electrophoresis showed that the one-armed anti-c-Met antibody 2E6 had a light chain $(\sim 30 \mathrm{kDa})$ and a heavy chain $(\sim 53$ $\mathrm{kDa})$, which were in accordance with the parental antibody 1H9D6 (Figure 1B, lane 1), and Fc ( $\sim 35 \mathrm{kDa})$ (Figure 1B, lane 2). The larger molecular weight (MW) of the light chain and $\mathrm{Fc}$ than that was calculated from the protein sequence ( 24 $\mathrm{kDa}$ for the light chain and $25 \mathrm{kDa}$ for $\mathrm{Fc}$ ) indicated that the eukaryotic expression preserved the glycosylation of the antibody. Non-denaturing conditions result in a great error in the protein marker designed mainly for denaturing conditions. Hence, in the non-denaturing polyacrylamide gel electrophoresis, the parental antibody 1H9D6 was chosen as an additional indicator to calibrate the error (Figure 1B, lane 1). The results showed that the difference between the MWs of 1H9D6 and 2E6 was $\sim 52 \mathrm{kDa}$, which was in accordance with that calculated from protein sequences. Taken together, the monovalent antibody $(\sim 102 \mathrm{kDa})$, redundant heavy chain $(\sim 53 \mathrm{kDa})$, and reduced level of the light chain $(\sim 24 \mathrm{kDa})$ were detected for 2E6 (Figure 1B, lane 2).

The binding of the one-armed anti-c-Met antibody to the target was further detected. Like the parental antibody 1H9D6, 2E6 bound to immobilized c-Met ECD-His in a dose-dependent manner (Figure 1C). The EC50 was $0.2716 \mathrm{nM}$ for $1 \mathrm{H} 9 \mathrm{D} 6$ and $1.758 \mathrm{nM}$ for $2 \mathrm{E} 6$. The variability likely resulted from the difference in the complementarity-determining region $(\mathrm{CDR}) / \mathrm{MW}$ ratio. In a cell line HepG2 with the high expression of c-Met, the similar binding capacity of 2E6 and 1H9D6 was confirmed by flow cytometry analysis (Figure 1D).

To detect the specificity of 2E6, a full length Western blot was conducted with 2E6 on the lysates from (CHO), Henrietta Lacks (Hela), and Hep G2. The results showed the strong bands at $\sim 135 \mathrm{kDa}$ for the mature c-Met $\beta$ subunit and the weaker bands at $\sim 100 \mathrm{kDa}$ for the underglycosylated c-Met in the blots of c-Met high-expressing 
A

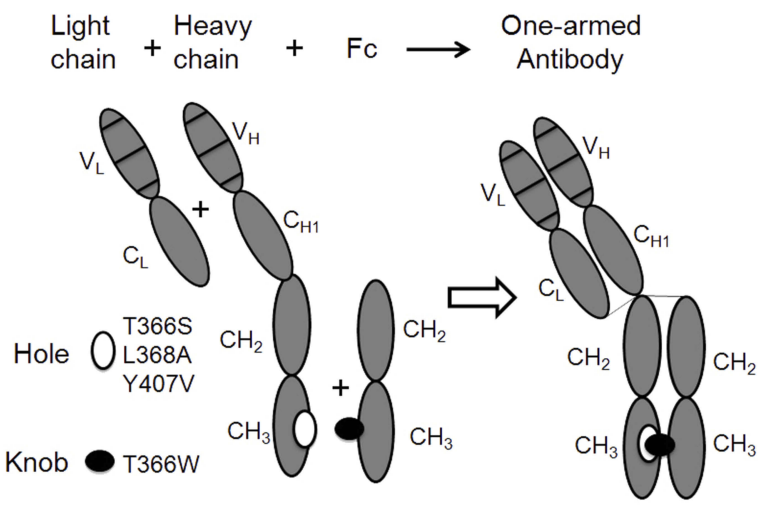

C

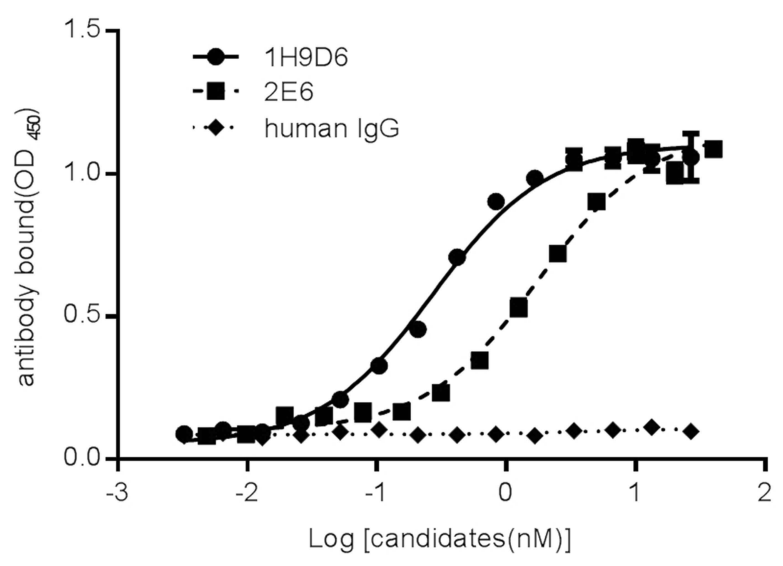

B

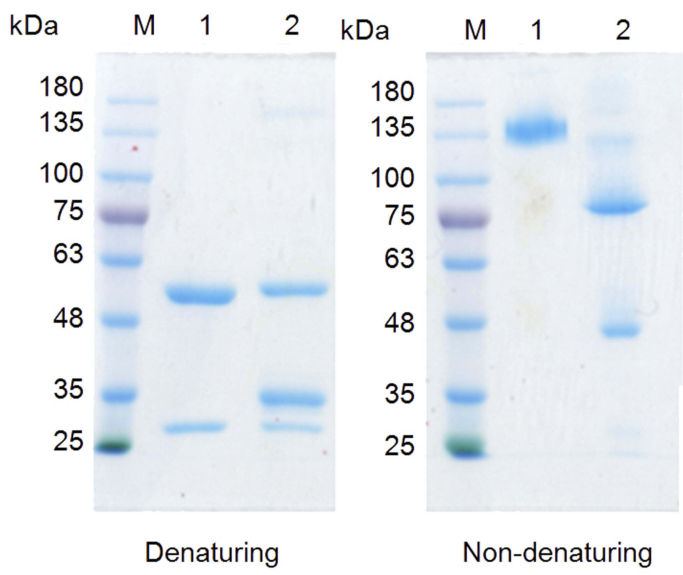

D

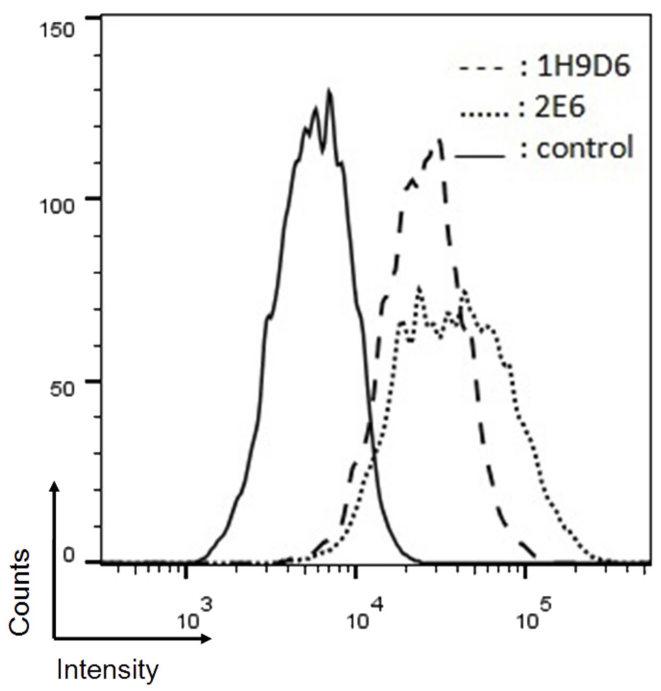

Figure I Generation and characterization of the one-armed anti-c-Met antibody.

Notes: (A) Schematic of the design of a one-armed monovalent antibody. (B) Denaturing and nondenaturing PAGE analysis of the full-length bivalent anti-c-Met antibody IH9D6 (lane I) and the monovalent one-armed anti-c-Met antibody 2E6 (lane 2). (C) Binding of IH9D6 (triangle), 2 E6 (square), and a human IgG (diamond) to the immobilized c-Met ECD-His at the indicated concentrations. Results were the averages of duplicate from the representative of three independent experiments, error bars represent the SD. (D) Binding of IH9D6 or 2E6 to HepG2 cells with the high expression of c-Met was assessed by flow cytometry. The chain line dash indicates the reactivity of IH9D6 at 13.3nM, the dot line dash indicates the reactivity of $2 \mathrm{E} 6$ at $20 \mathrm{nM}$, and the solid line indicates the reactivity of a control antibody at $20 \mathrm{nM}$.

Abbreviations: c-Met, c-mesenchymal-epithelial transition factor; PAGE, polyacrylamide gel electrophoresis; ECD, extracellular domain; SD, standard deviation.

cell lines, Hela and Hep G2. For the CHO blots, no obvious bands were detected with 2E6 (Figure 2A). These results indicated the low expression level of c-Met in $\mathrm{CHO}$ or the low binding affinity of 2E6 to Chinese hamster c-Met. Hence, $\mathrm{CHO}$ was employed to validate the specificity of 2E6 in living cells. The transient expression of human c-Met fragment comprising the extracellular domain and the trans-membrane domain (prrl-c-Met$\mathrm{TM}$ ) on the $\mathrm{CHO}$ surface caused the interaction of 2E6 with CHO-c-Met-TM in a dose-dependent manner in the flow cytometric analysis (Figure 2B). The RNAi knock down of c-Met was further conducted in Hep G2. As expected, the knock down of c-Met caused the reduction of binding affinity of 2E6 to Hep G2-shc-Met in the flow cytometric analysis (Figure 2C).

\section{One-Armed Anti-c-Met Antibody Reduced the HGF/c-Met Signaling Axis}

A one-armed anti-c-Met antibody, derived from a full-length antibody whose epitope overlapped with the HGF-binding site, was designed to antagonize the $\mathrm{HGF} / \mathrm{c}-$ Met signaling axis by blocking the HGF/c-Met interaction. 2 E6 was first tested for its ability to compete with HGF in a ligand-binding assay. As expected, 2E6 inhibited the recombinant HGF 
A

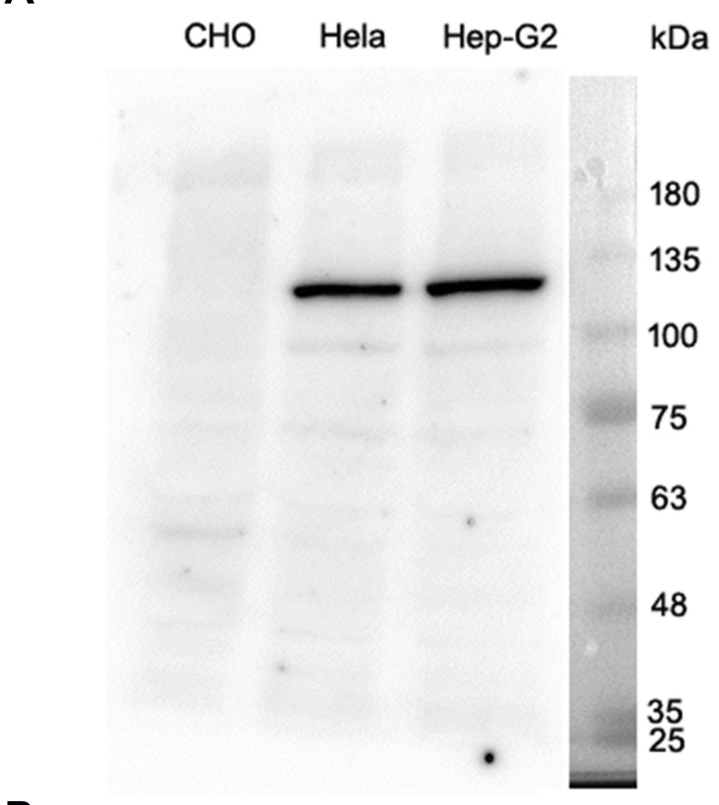

B

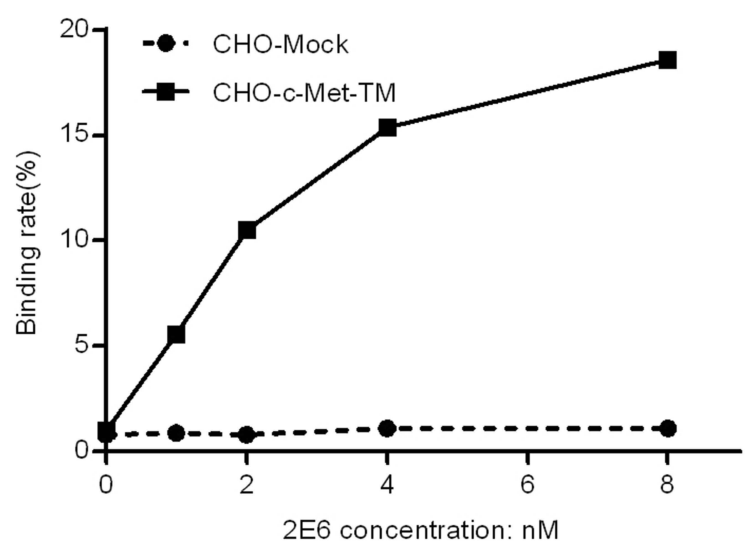

C

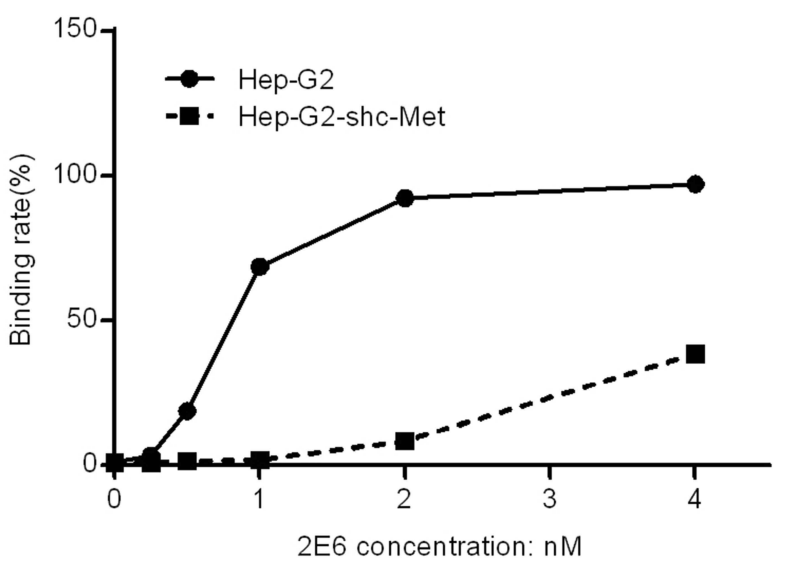

Figure 2 The specificity of the one-armed anti-c-Met antibody to the target.

Notes: (A) The full length Western blot conducted with 2E6 on the lysates from Chinese hamster ovary cells (CHO), Henrietta Lacks (Hela) and Hep G2. (B) The effect of the high expression of c-Met-TM on the binding of 2E6 to CHO. Prrl-c-Met-TM or mock transfected CHO cells were treated with $2 \mathrm{E} 6$ at indicated concentrations and assessed by flow cytometry. The ratios of the positive cells versus the total cells were calculated. (C) The effect of RNAi knock down of c-Met on the binding of $2 \mathrm{E} 6$ to Hep G2. Prrl-shc-Met or mock transfected Hep G2 cells were treated with 2E6 at the indicated concentrations and assessed by flow cytometry. The ratios of the positive cells versus the total cells were calculated.

Abbreviations: c-Met, c-mesenchymal-epithelial transition factor; $\mathrm{CHO}$, Chinese hamster ovary cells; RNAi, RNA interference. 
binding to immobilized Met ECD-HIS in a dose-dependent mode, with an EC50 value of 4.326nM. Similar results were obtained with the parental antibody 1H9D6, with an EC50 value of $2.159 \mathrm{nM}$ (Figure $3 \mathrm{~A}$ ). To confirm the competitive activity of 2E6 with HGF in living cells, the influence of HGF $(10 \mathrm{nM})$ on the reactivity of 2E6 (20nM) to the cell line HepG2 with the high expression of c-Met was examined by flow cytometry analysis. The presence of HGF resulted in a significant decrease in the reactivity of HepG2 with 2E6 (Figure 3B). To detect the effect of 2E6 on the HGF-induced

A

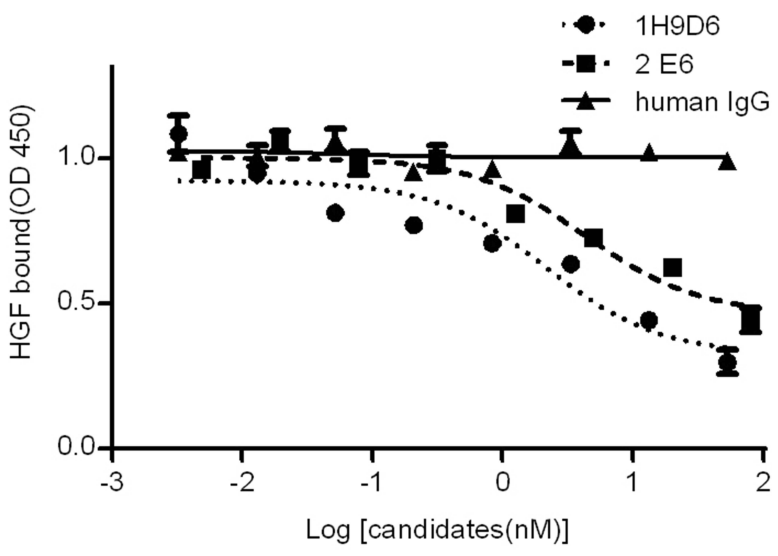

B

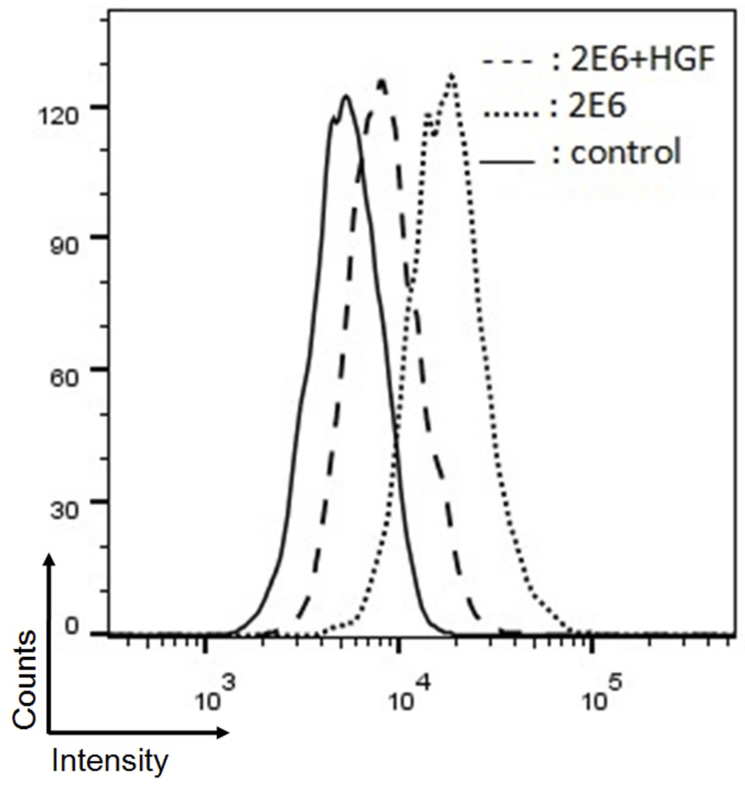

Figure 3 The competitive inhibition of the one-armed anti-c-Met antibody against HGF binding to c-Met.

Notes: (A) The binding of HGF ( InM) to immobilized c-Met ECD-His in the presence of IH9D6 (black circle), 2E6 (black square), or human lgG (black triangle) at the indicated concentrations. Results were the averages of duplicate from the representative of three independent experiments, error bars represent the SD. (B) The binding of 2E6 (20nM) with (chain line dash) or without (dot line dash) HGF (IOnM) to HepG2 cells with the high expression of c-Met was assessed by flow cytometry. The solid line indicates the reactivity of a control antibody at $66.7 \mathrm{nM}$.

Abbreviations: c-Met, c-mesenchymal-epithelial transition factor; HGF, hepatocyte growth factor; ECD, extracellular domain; IgG, Immunoglobulin G; SD, standard deviation. phosphorylation of c-Met and downstream signaling pathways, the cellular kinase-targeting activity of HGF was verified in HepG2 cells. A time course of c-Met, Gab-1, Akt, and Erk1/2 phosphorylation after incubation with HGF $(50 \mathrm{ng} / \mathrm{mL})$ was detected. The results indicated a rapid and transient increase in $\mathrm{HGF} / \mathrm{c}-\mathrm{Met}$ signaling, and the maximal phosphorylation appeared in 5 min after HGF treatment (Figure 4A). Pretreatment with 2E6 (10nM) and a c-Met/ anaplastic lymphoma kinase (ALK) tyrosine kinase inhibitor (TKI) PF-02341066 (1 $\mu \mathrm{M})$, which was used as positive control, caused a dramatic decrease in the c-Met, Gab-1, Akt, and Erk1/2 phosphorylation 5 min after HGF (0.5nM) stimulation at the same level (Figure 4B). These results indicated that the cellular kinase-targeting activity could be inhibited by antagonizing the HGF/c-Met interaction and inhibiting tyrosine kinase activity withone-armed anti-c-Met antibody.

\section{Antitumor Activity of the One-Armed Anti-c-Met Antibody in HCC Cell Lines}

The effect of 2E6 on the growth of HCC cell line HepG2 was investigated. In both HGF-treated or non-HGF-treated cells, 2E6 (1-25nM) significantly inhibited cell growth in a dosedependent manner (Figure 5A). Interestingly, treatment with exogenous HGF exerted a little effect on cell growth and the inhibition rate of 2E6. These results indicated that the onearmed anti-c-Met antibody most likely inhibited HepG2 proliferation by interfering with the HGF autocrine loop. The effect of 2E6 on the migration and invasion of a panel of HCC cell lines, including, HepG2, Huh-7, Hep 3B2 and SMMC-7721 cells was further investigated in a Transwell assay. In contrast to cell growth, the migration and invasion of HCC cells were strongly stimulated by exogenous HGF, and dramatically suppressed by $2 \mathrm{E} 6(1-25 \mathrm{nM})$ treatment (Figure 5B). When the 2E6 concentration was more than $5 \mathrm{nM}$, the number of migrated Hep G2 cells decreased even below the basal level. These results indicated that both exogenous and endogenous HGF-induced cell migration in $\mathrm{HCC}$ cells could be inhibited by $2 \mathrm{E} 6$.

\section{One-Armed Anti-c-Met Antibody Reduced HGF-Induced Angiogenesis}

The activation of c-Met plays the most crucial role in regulating tumor angiogenesis. Because HGF-induced c-Met activation eventually stimulates endothelial cell responses, 2E6 was tested for its ability to inhibit HGFinduced HUVEC responses. In the case of endothelial cell proliferation, a cell viability assay was conducted. HGF 
A

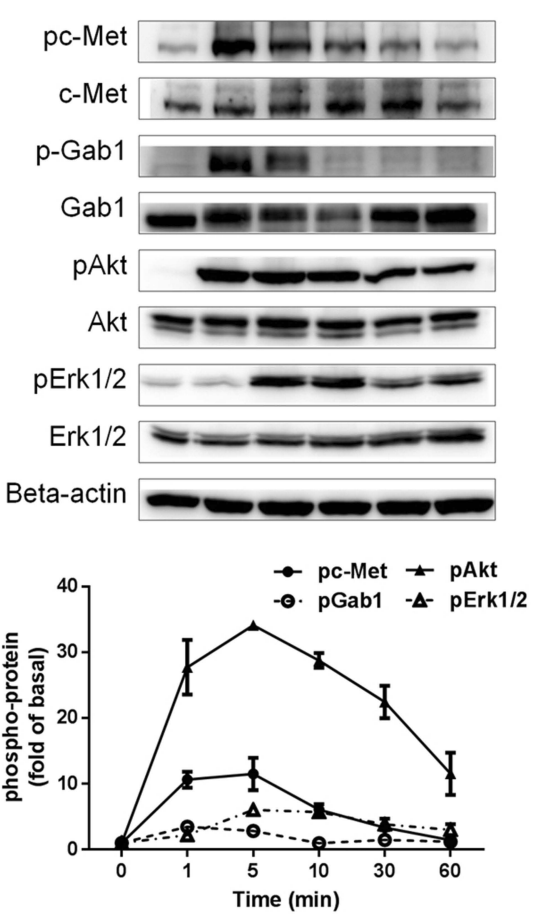

B

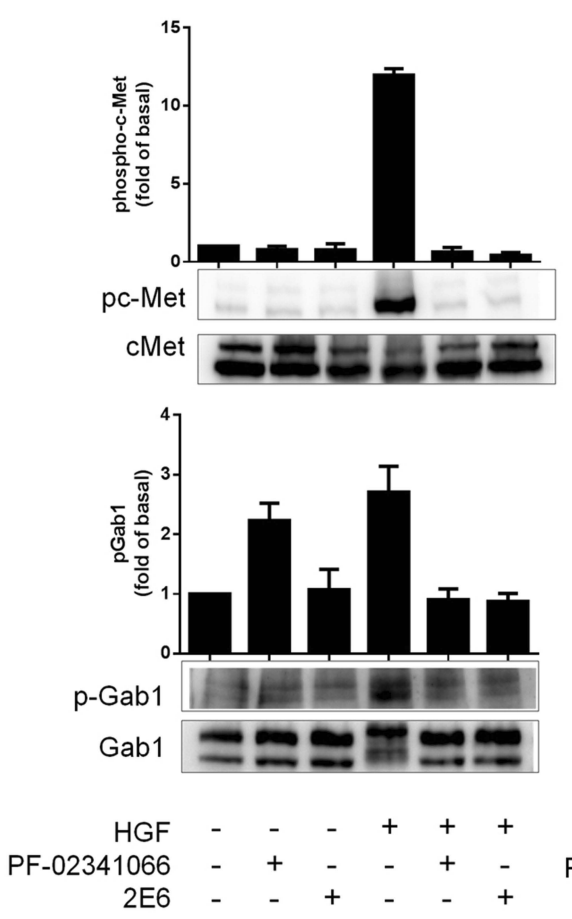

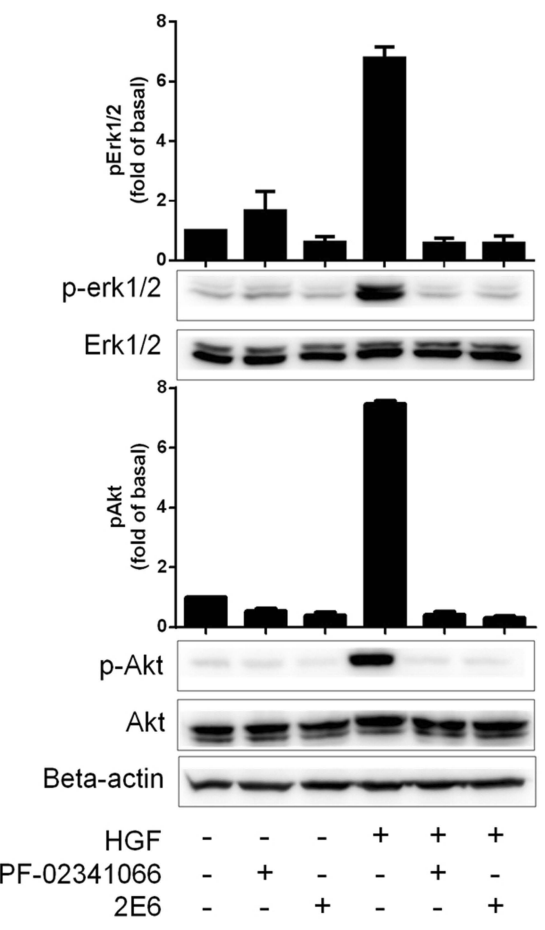

Figure 4 The inhibition of the one-armed anti-c-Met antibody on the HGF/c-Met axis.

Notes: (A) The time course of c-Met, Gab-I, Akt, and Erk phosphorylation in HepG2 cells after incubation with HGF (InM). (B) Cells were treated with 2E6 (IOnM) or PF$02341066(\mathrm{I} \mu \mathrm{M})$ for $120 \mathrm{~min}$ before stimulation with HGF (InM) for 5 min. c-Met, Gab-I, Akt, and Erk phosphorylation levels were measured using immunoblotting. The results were the averages of duplicate from the representative of three independent experiments, the error bars represent the SD.

Abbreviations: c-Met, c-mesenchymal-epithelial transition factor; HGF, hepatocyte growth factor; Gab-I, Grb2-associated binding protein I; Akt, v-Akt gene expression protein, also referred to as PKB (Protein kinase B); Erk, Extracellular regulated protein kinases; PF-02341066: Crizotinib, a c-Met inhibitor; SD, standard deviation.

$(0.5 \mathrm{nM})$ significantly improved HUVEC viability, while 2E6 (2.5-20nM) inhibited HGF-induced HUVEC proliferation in a dose-dependent manner (Figure 6A). In the case of endothelial cell tube formation, an in vitro Matrigel angiogenesis assay was conducted. HGF-induced HUVEC tube formation $(0.5 \mathrm{nM})$ dramatically increased compared with the control, while 2E6 (2.5-10nM) inhibited HGFinduced HUVEC tube formation (Figure 6B).

\section{One-Armed Anti-c-Met Antibody Inhibited HepG2 Tumor Xenograft Growth}

The anticancer activity of 2E6 was evaluated in HepG2 xenografts in mice within 6 weeks of $2 \mathrm{E} 6$ injection. As shown in Figure 7A, the administration of 2E6 delayed the growth of HepG2 cells. 2E6 treatment dramatically inhibited xenograft tumor volumes (Figure 7B). Further, $5 \mathrm{mg} / \mathrm{kg}$ of 2E6 caused $44.5 \% \pm 13.3 \%$ inhibition of tumor volumes, and $10 \mathrm{mg} / \mathrm{kg}$ of $2 \mathrm{E} 6$ caused $65.8 \% \pm 12.5 \%$ inhibition of tumor volumes. The mouse body weights were almost unchanged between the groups, indicating that the administration of 2E6 was generally safe for the experimental mice (data not shown).

\section{Discussion}

C-Met and its ligand HGF are widely expressed and precisely regulated in normal tissues. In HCC, paracrine delivered HGF, autocrine loop formation, and overexpression of ligand and/or receptor cause inappropriate c-Met signaling. The upregulated c-Met pathway promotes aggressive oncogenic phenotypes, including cell migration and invasion capability, proliferation, and tumor angiogenesis. The present study showed that c-Met inhibition by a novel one-armed anti-c-Met antibody suppressed the oncogenic phenotypes of HCC in vitro and in vivo. These results indicated that the antibody targeted at the receptor might be able to overcome the overexpression of ligand and/or receptor in tumor tissues.

The inhibition of HGF/c-Met has been identified as a potential therapy for HCC. Several types of agents are under development for the c-MET signaling inhibitors, including HGF-neutralizing antibodies, HGF antagonists, 


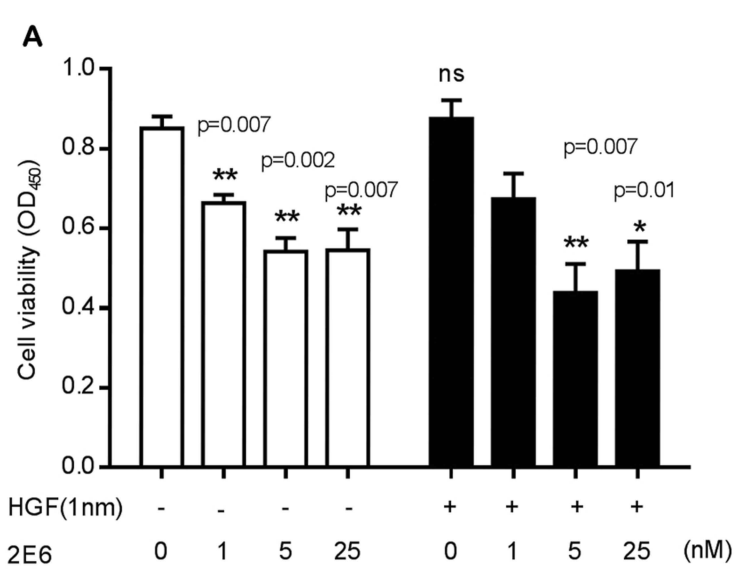

B
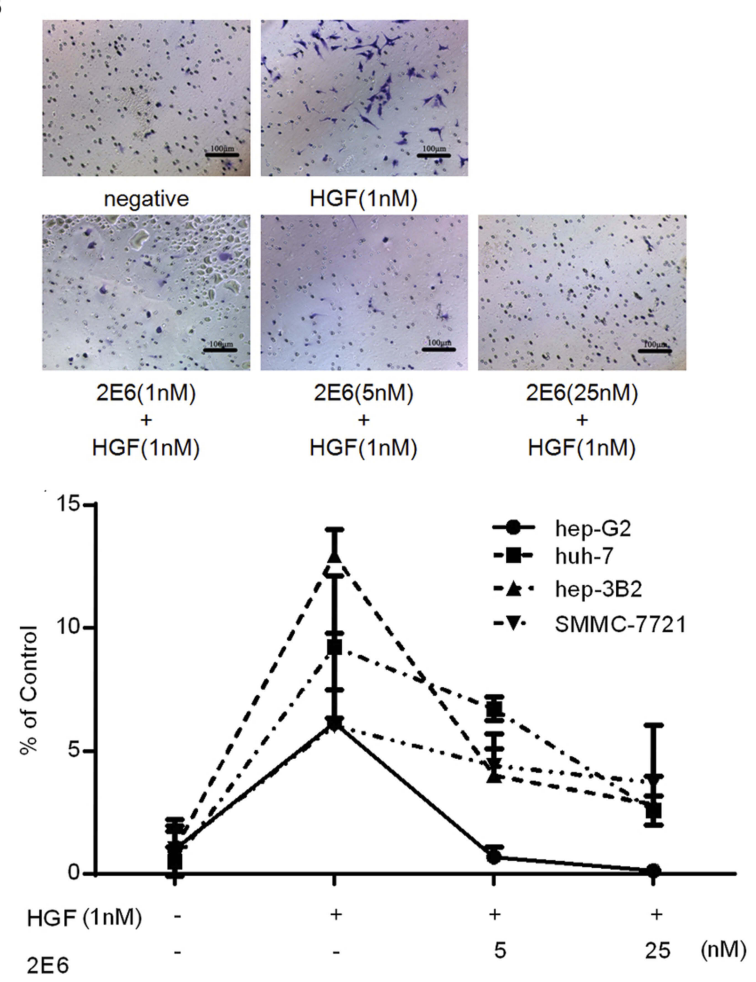

Figure 5 The antitumor activity of $2 \mathrm{E} 6$ in the $\mathrm{HCC}$ cells.

Notes: (A) The effect of the incubation for $72 \mathrm{~h}$ with HGF $(0.1 \mathrm{InM})$ or $2 \mathrm{E} 6(\mathrm{I}-25 \mathrm{nM})$ at the indicated concentration. The cell line was analyzed using the Cell Counting Kit CCK8NST-8 cell viability assay. Error bars indicate the standard error of the mean. For all results, ${ }^{*} \mathrm{P}<0.05$, ${ }^{* *} \mathrm{P}<0.01$, and, versus the $2 \mathrm{E} 6$ nontreated groups in the presence or absence of HGF; ns, nonsignificance, versus the control group. (B) The representative photographs of the Transwell assay show the HepG2 cells that penetrated through the Transwell. The images were captured using an inverted microscope with a $\times 10$ objective (upper panel). The statistical analysis of the numbers of Hep G2, Huh-7, Hep 3B2 and SMMC-772 I migrating to the bottom chambers. Results were the averages of duplicate from the representative of three independent experiments, error bars represent the SD. The statistical analysis of the Transwell assay showed a significantly lower number of migrated cells. (lower panel).

Abbreviations: HGF, hepatocyte growth factor; WST-8 (2-(4-lodophenyl)3-(4-nitrophenyl)-5-(2,4-disulfophenyl)-2H-tetrazolium, monosodium salt); HCC, hepatocellular carcinoma.

and c-Met TKIs. However, for these inhibitors, especially for HGF antagonists and TKIs, the off-target activity, toxicity, and variation of the tumor c-Met status (gene mutation, gene amplification, and overexpression) often result in failure in clinical trials. Thus, highly selective antibodies are expected to overcome these problems. In the last few years, multiple antibody-based strategies were used to inhibit the HGF/c-Met axis. Anti-HGF antibodies that blocked the binding of human HGF to c-Met were investigated. Anti-HGF antibodies were proved to inhibit HGF-mediated c-Met phosphorylation, cell proliferation, survival, and invasion in vitro. These antibodies inhibited HGF-dependent autocrine-driven tumor growth and caused the significant regression of established U-87 MG tumor xenografts. ${ }^{18}$ Meanwhile, approaches to develop inhibitory anti-c-Met monoclonal antibody have encountered a number of difficulties. Epitope difference may account for the different functional agonist properties of the anti-MET antibody. Only a few full-length and bivalent anti-MET monoclonal antibodies were reported to have an inhibitory effect. For example, R13, R28, and LY2875358 were reported to inhibit the $\mathrm{HGF} / \mathrm{c}-$ Met interaction and the subsequent activation of c-Met, Akt, and Erk1/2, as well as HGF-induced migration and proliferation. ${ }^{19,20}$ Most c-Met antibodies have intrinsic agonistic activity resulting from their bivalent structure, which induces the dimerization and autophosphorylation of RTKs in a ligandindependent manner. The reconstruction of the anti-c-Met antibody to build a monovalent one-armed antibody is a widely accepted strategy to overcome the agonistic activity of anti-c-Met.

Recombinant human single-chain variable fragments $(\mathrm{scFv})$ have been developed against various therapeutic targets for cancer treatments. ${ }^{21}$ The specific binding of anti-c-Met scFv to c-Met can cause growth inhibition and induction of apoptosis pathways in tumor cells in vitro, ${ }^{22-24}$ but its short half-life limits its application in vivo. To overcome this problem, the knobs-into-holes technology was adopted to construct a one-armed anti-c-Met antibody with a long half-life. In the last few years, Genentech (CA, USA) has developed a recombinant humanized monoclonal monovalent anti-c-Met antibody onartuzumab (OA5D5, MetMAb) and investigated its preclinical activity in glioblastoma (GBM), pancreatic cancer, breast cancer, and nonsmall-cell lung cancer (NSCLC). However, no study explored the effect of the one-armed anti-c-Met antibody on HCC.

In this study, a novel one-armed anti-c-Met antibody 2E6 was constructed, which had similar affinity to c-Met as that of onartuzumab, to confirm the antitumor activity of the monovalent anti-c-Met antibody in HCC. As the 


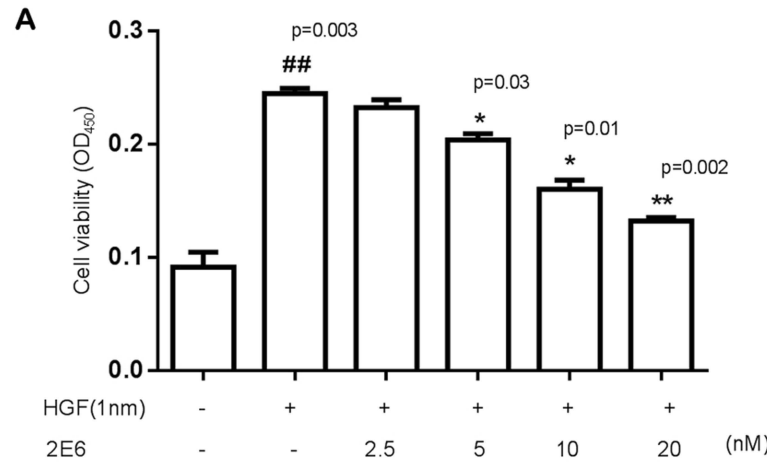

B
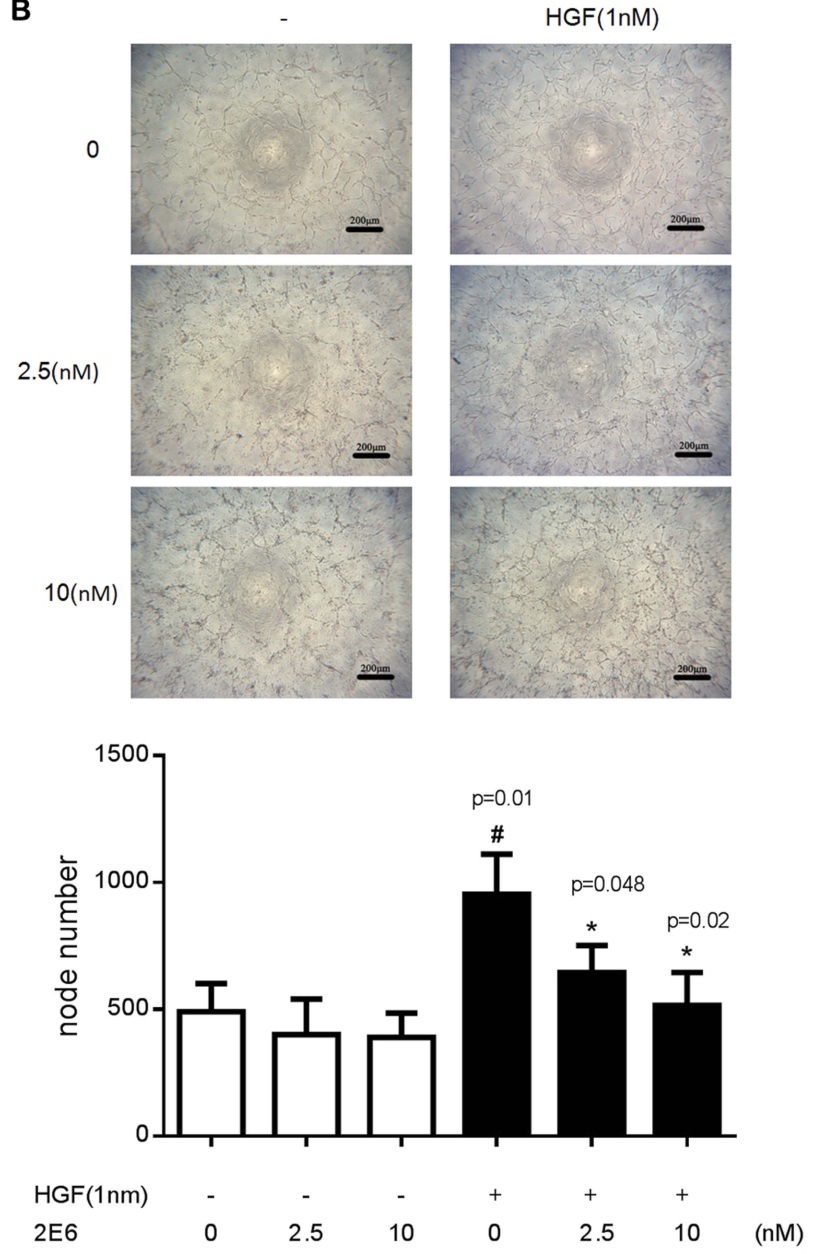

Figure 6 Inhibitory effect of the one-armed anti-c-Met antibody on angiogenesis. Notes: (A) The effect of incubation for $72 \mathrm{~h}$ with HGF or $2 \mathrm{E} 6$ at the indicated concentration. The cell line was analyzed using the Cell Counting Kit CCK-8/ WST-8 cell viability assay. Error bars indicate the standard error of the mean. ${ }^{\#} \mathrm{P}<0.01$ versus the control group; $* \mathrm{P}<0.05$, $* * \mathrm{P}<0.01$ versus the HGFtreated groups. (B) $2 \mathrm{E} 6$ suppressed the vascular endothelial cell tube formation on 3D Matrigel. HUVECs were seeded into a 96-well plate pre-coated with Matrigel and incubated with HGF and 2E6 at the indicated concentrations for 18 h. The images were captured using an inverted microscope with a $\times 4$ objective (upper panel). The statistical analysis of the node numbers were counted by Imagej. Results were the averages of duplicate from the representative of three independent experiments. Error bars represent the SD. \#P $<0.01$ versus the control group; *P $<0.05$ versus the HGF-treated groups (lower panel).

Abbreviations: HGF, hepatocyte growth factor; WST-8 (2-(4-lodophenyl)-3-(4-nitrophenyl)-5-(2,4-disulfophenyl)-2H-tetrazolium, monosodium salt); HUVEC, human umbilical vein endothelial cells.
A

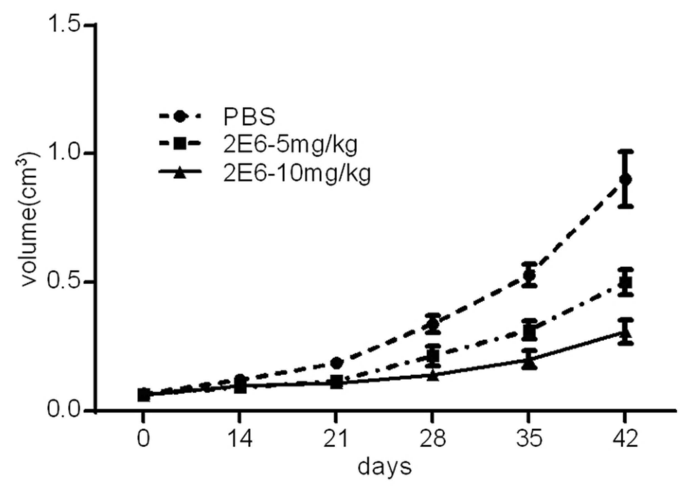

B PBS

$2 \mathrm{E} 6(5 \mathrm{mg} / \mathrm{kg})$
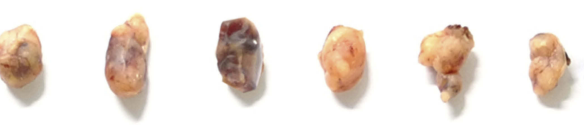

2E6 $(10 \mathrm{mg} / \mathrm{kg})$

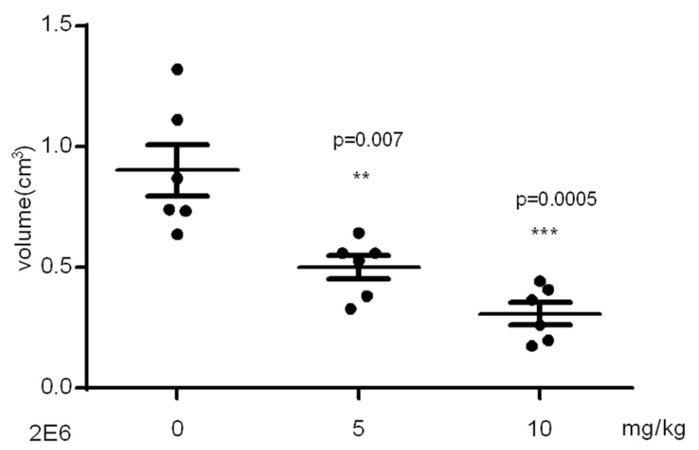

Figure 7 The inhibitory effect of 2E6 on HepG2 tumor xenograft growth.

Notes: (A) The BALB/c nude mice ( $n=6$ per group) bearing HepG2 xenograft tumors were treated with either the vehicle or 2E6 at a dose of 5 and $10 \mathrm{mg} / \mathrm{kg}$ (intraperitoneally, twice weekly). The data are shown as group mean tumor volumes, with the standard error of the mean indicated. (B) The tumor measurement after the mice were sacrificed (day 42 after the first intraperitoneal injection). The bars indicate mean \pm standard deviation. ${ }^{* * P}<0.01$, ***P $<0.001$ versus the PBS-treated group.

$\mathrm{HGF} / \mathrm{c}-$ Met axis is a key pathway in HCC, the antagonistic activity of 2E6 was first detected based on the c-Met signaling pathway. The HGF/c-Met axis usually comprises HGF binding, c-Met dimerization and phosphorylation, activation of adaptor proteins, and subsequent multiple signaling pathways, among which PI3K/ Akt and Ras/Erk-MAPK contribute to HGF-induced invasion and migration. ${ }^{25}$ In this study, the time course of the phosphorylation of c-Met, Gab-1, Erk, and Akt after HGF stimulation was verified in an HCC cell line HepG2, which was then used to investigate the influence 
of $2 \mathrm{E} 6$ on the HGF/c-Met axis. A c-Met/ALK TKI, PF02341066 (Crizotinib), was chosen as a positive control. The results showed that the one-armed anti-c-Met antibody markedly inhibited the HGF/c-Met signaling pathway to the same extent as TKI. Multiple antitumor effects of this antibody were further investigated in HepG2 cells. Taken together, the results suggested that 2E6 had the antiproliferative capacity that contributed to the inhibition of tumor growth and antimigratory effects in HCC.

Another mechanism that seems to have contributed to the antitumor effect of the 2E6 antibody is the inhibition of tumor angiogenesis. The HGF-induced proliferation and tube formation of HUVECs were reduced by 2E6 treatment. The signaling through c-Met is known to directly mediate proangiogenic effects, such as endothelial cell migration, proliferation, and formation of three-dimensional tubes. The interruption of HGF/c-Met signaling likely also accounts for the inhibitory effect of the one-armed anti-c-Met antibody on tumor vasculature.

Although the antitumor activity of anti-c-Met antibodies was proved by researchers, the development of the antibody-based c-Met inhibitor is still controversial. Apart from the HGF-dependent activation, non-HGF factors can cause c-Met activation, such as overexpression-induced activation, mutation-induced activation, or transactivation of other receptors. Most anti-c-Met antibodies were developed as potential therapeutics antagonizing HGF binding and therefore inhibiting the HGF-dependent, but not the non-HGF-dependent, c-Met activity. Some of the results of the present study suggested that the c-Met antibody might inhibit non-HGF-induced c-Met activation. (1) In the absence of exogenous HGF, 2E6 still inhibited HepG2 proliferation (Figure 4A). (2) With the increasing concentration of 2E6, HepG2 migration decreased even below the basal level (Figure 4B). These phenomena might be explained by interfering the autocrine loop of HGF. However, while further investigating the transcription level of HGF in cultured HepG2 cells, the real-time PCR showed much lower HGF mRNA level compared with the c-Met mRNA level (data not shown). Hence, it was speculated that the basal c-Met activity in HepG2 cells might also originate from non-HGF factors and the diverse epitopes on antigen surface might offer the opportunity for different monoclonal anti-c-Met antibodies to mask the interface between c-Met and activating factors in an HGFdependent or a non-HGF-dependent manner.

\section{Conclusion}

In conclusion, the present study confirmed that activated c-Met was one of the promising targets of anticancer drugs. The monovalent anti-c-Met antibody is an attractive strategy of drug development for c-Met inhibition, which has the potential to overcome the intrinsic agonistic activity of the full-length bivalent anti-c-Met antibody.

\section{Acknowledgment}

This study was financially supported by the National Major Scientic and Technological Special Project for Significant New Drugs Development (2019ZX09732002-12), the Natural Science Foundation of Jiangsu Province of China (BK20150278) and the Shanghai Science and Technology Commission (19431903200).

\section{Disclosure}

Jianmin Fang reports personal fees from RemeGen Ltd., outside the submitted work. The authors report no other conflicts of interest in this work.

\section{References}

1. Ferlay J, Soerjomataram I, Dikshit R, et al. Cancer incidence and mortality worldwide: sources, methods and major patterns in GLOBOCAN 2012. Int $J$ Cancer. 2015;136(5):E359-E386. doi:10.1002/ijc. 29210

2. Grandhi MS, Kim AK, Ronnekleiv-Kelly SM, Kamel IR, Ghasebeh MA, Pawlik TM. Hepatocellular carcinoma: from diagnosis to treatment. Surg Oncol. 2016;25(2):74-85. doi:10.1016/j.suronc.2016.03.002

3. Nakamura T, Nishizawa T, Hagiya M, et al. Molecular cloning and expression of human hepatocyte growth factor. Nature. 1989;342 (6248):440-443. doi:10.1038/342440a0

4. Lokker NA, Mark MR, Luis EA, et al. Structure-function analysis of hepatocyte growth factor: identification of variants that lack mitogenic activity yet retain high affinity receptor binding. EMBO J. 1992;11 (7):2503-2510. doi:10.1002/embj.1992.11.issue-7

5. Naka D, Ishii T, Yoshiyama Y, et al. Activation of hepatocyte growth factor by proteolytic conversion of a single chain form to a heterodimer. J Biol Chem. 1992;267(28):20114-20119.

6. Peruzzi B, Bottaro DP. Targeting the c-Met signaling pathway in cancer. Clin Cancer Res. 2006;12(12):3657-3660. doi:10.1158/10780432.CCR-06-0818

7. Eckerich C, Zapf S, Fillbrandt R, Loges S, Westphal M, Lamszus K. Hypoxia can induce c-Met expression in glioma cells and enhance SF/ HGF-induced cell migration. Int $J$ Cancer. 2007;121(2):276-283. doi:10.1002/(ISSN)1097-0215

8. Hara S, Nakashiro K, Klosek SK, Ishikawa T, Shintani S, Hamakawa H. Hypoxia enhances c-Met/HGF receptor expression and signaling by activating HIF-1alpha in human salivary gland cancer cells. Oral Oncol. 2006;42(6):593-598. doi:10.1016/j.oraloncol ogy.2005.10.016

9. Ueki T, Fujimoto J, Suzuki T, Yamamoto H, Okamoto E. Expression of hepatocyte growth factor and its receptor, the c-met proto-oncogene, in hepatocellular carcinoma. Hepatology. 1997;25(3):619-623. doi:10.1002/(ISSN)1527-3350 
10. Cecchi F, Rabe DC, Bottaro DP. Targeting the HGF/Met signalling pathway in cancer. Eur $J$ Cancer. 2010;46(7):1260-1270. doi:10.1016/j.ejca.2010.02.028

11. Bouattour M, Raymond E, Qin S, et al. Recent developments of c-Met as a therapeutic target in hepatocellular carcinoma. Hepatology. 2018;67(3):1132-1149. doi:10.1002/hep.v67.3

12. Surati M, Patel P, Peterson A, Salgia R. Role of MetMAb (OA-5D5) in c-MET active lung malignancies. Expert Opin Biol Ther. 2011;11 (12):1655-1662. doi:10.1517/14712598.2011.626762

13. Prat M, Crepaldi T, Pennacchietti S, Bussolino F, Comoglio PM. Agonistic monoclonal antibodies against the Met receptor dissect the biological responses to HGF. J Cell Sci. 1998;111(Pt 2):237-247.

14. Ohashi K, Marion PL, Nakai H, et al. Sustained survival of human hepatocytes in mice: a model for in vivo infection with human hepatitis B and hepatitis delta viruses. Nat Med. 2000;6(3):327-331. doi:10.1038/ 73187

15. Martens T, Schmidt NO, Eckerich C, et al. A novel one-armed anti-c-Met antibody inhibits glioblastoma growth in vivo. Clin Cancer Res. 2006;12 (20 Pt 1):6144-6152. doi:10.1158/1078-0432.CCR-05-1418

16. Jin $\mathrm{H}$, Yang $\mathrm{R}$, Zheng $\mathrm{Z}$, et al. MetMAb, the one-armed 5D5 anti-c-Met antibody, inhibits orthotopic pancreatic tumor growth and improves survival. Cancer Res. 2008;68(11):4360-4368. doi:10.1158/0008-5472.CAN-07-5960

17. Merchant M, Ma X, Maun HR, et al. Monovalent antibody design and mechanism of action of onartuzumab, a MET antagonist with anti-tumor activity as a therapeutic agent. Proc Natl Acad Sci U S A. 2013;110(32):E2987-E2996. doi:10.1073/pnas.1302725110

18. Burgess T, Coxon A, Meyer S, et al. Fully human monoclonal antibodies to hepatocyte growth factor with therapeutic potential against hepatocyte growth factor/c-Met-dependent human tumors. Cancer Res. 2006;66(3):1721-1729. doi:10.1158/0008-5472.CAN05-3329
19. van der Horst EH, Chinn L, Wang M, et al. Discovery of fully human anti-MET monoclonal antibodies with antitumor activity against colon cancer tumor models in vivo. Neoplasia. 2009;11(4):355-364. doi: $10.1593 /$ neo. 81536

20. Liu L, Zeng W, Wortinger MA, et al. LY2875358, a neutralizing and internalizing anti-MET bivalent antibody, inhibits HGF-dependent and HGF-independent MET activation and tumor growth. Clin Cancer Res. 2014;20(23):6059-6070. doi:10.1158/1078-0432.CCR-14-0543

21. Tao J, Xiang JJ, Li D, Deng N, Wang H, Gong YP. Selection and characterization of a human neutralizing antibody to human fibroblast growth factor-2. Biochem Biophys Res Commun. 2010;394 (3):767-773. doi:10.1016/j.bbrc.2010.03.067

22. Liu Z, Feng Z, Zhu X, et al. Construction, expression, and characterization of an anti-tumor immunotoxin containing the human anti-c-Met single-chain antibody and PE38KDEL. Immunol Lett. 2013;149(1-2):30-40. doi:10.1016/j.imlet.2012.09.006

23. Lu RM, Chang YL, Chen MS, Wu HC. Single chain anti-c-Met antibody conjugated nanoparticles for in vivo tumor-targeted imaging and drug delivery. Biomaterials. 2011;32(12):3265-3274. doi:10.1016/j.biomaterials.2010.12.061

24. Qamsari ES, Sharifzadeh Z, Bagheri S, et al. Isolation and characterization of anti c-met single chain fragment variable $(\mathrm{scFv})$ antibodies. $J$ Immunotoxicol. 2017;14(1):23-30. doi:10.1080/ 1547691X.2016.1251512

25. Zhou HY, Wong AS. Activation of p70S6K induces expression of matrix metalloproteinase 9 associated with hepatocyte growth factor-mediated invasion in human ovarian cancer cells. Endocrinology. 2006;147(5):2557-2566. doi:10.1210/en.2005-1404

\section{Publish your work in this journal}

Drug Design, Development and Therapy is an international, peerreviewed open-access journal that spans the spectrum of drug design and development through to clinical applications. Clinical outcomes, patient safety, and programs for the development and effective, safe, and sustained use of medicines are a feature of the journal, which has also been accepted for indexing on PubMed Central. The manuscript management system is completely online and includes a very quick and fair peer-review system, which is all easy to use. Visit http://www. dovepress.com/testimonials.php to read real quotes from published authors. 